Université de Strasbourg

Séminaire de Probabilités

1975/76

\title{
UN POINT DE PRIORITE
}

P.A.Meyer

Il est vrai que les questions de priorité ont peu d'importance en mathématiques, car nous serons bien vite oubliés, notre travail et nous mêmes. Mais puisque l'occasion se présente de réparer une petite injustice sans verser de sang, profitons en. Dans l'exposé "Questions de théorie des flots II " du séminaire IX, LAZARO et moi faisons remonter à HANEN (Annales Inst. H. Poincaré, 7, 1971) la découverte de l'identité entre les notions de mesure de PATM et de flot sous une fonction. On m'a signalé que cette remarque figurait explicitement dans un article un peu antérieur de F.PAPANGELOU "The Ambrose-Kakutani theorem and the Poisson process ", Contributions to Ergodic theory and Probability, Lecture Notes vol.160, 1970, p.234-240). 